\title{
A successful hybrid operation for life-threatening hemorrhagic shock secondary to an iatrogenic ruptured pseudoaneurysm of the external iliac artery following percutaneous arterial access
}

\author{
Takahiro Shoji, ${ }^{1}$ Hirohisa Harada, ${ }^{2}$ Shinji Yamazoe, ${ }^{3}$ Yoshihiro Yamaguchi ${ }^{1}$ \\ ${ }^{1}$ Department of Trauma and Critical Care Medicine, Kyorin University School of Medicine; ${ }^{2}$ Department of \\ Surgery, Tokyo Saiseikai Central Hospital; ${ }^{3}$ Department of Radiology, Tokyo Dental College Ichikawa \\ General Hospital, Tokyo, Japan
}

\begin{abstract}
Intravascular treatments such as arterial embolization and resuscitative endovascular balloon occlusion of the aorta are being increasingly performed in emergency cases, in addition to the increasing use of arterial access as an intensive care monitoring tool. Thus, arterial access-related complications are being commonly reported. A 40year-old man with renal artery stenosis underwent renal artery stent placement via the left inguinal puncture approach. After the procedure, his groin was manually compressed to hemostasis for $30 \mathrm{~min}$. He unexpectedly developed shock the following day, and computed tomography revealed a ruptured pseudoaneurysm of the left external iliac artery (EIA) following iatrogenic vascular trauma owing to an inappropriately performed groin puncture. We initially controlled the hemorrhage using endovascular balloon occlusion of the left EIA. Subsequently, the injured EIA was repaired using a direct suture. The postoperative course was uneventful. Herein, we evaluated the causes of iatrogenic complications and the effectiveness of our treatment strategy.
\end{abstract}

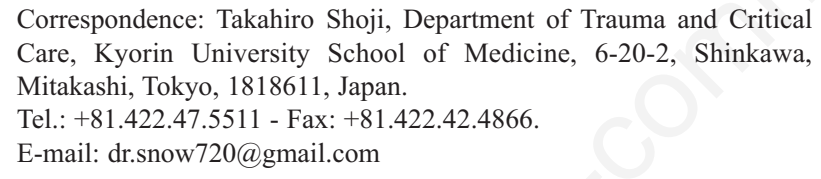

Key words: Vascular trauma; Endovascular treatment; Balloon occlusion.

Contributions: TS wrote the main text with advice from HH and YY. TS and SY collected the data and references. All authors discussed the case presentation, commented on the manuscript at all stages, read and approved the final manuscript for submission.

Conflict of interest: the authors declare no potential conflict of interest.

Funding: none.

Ethical statement: informed consent was obtained from the patient for publication of this case report and accompanying images.

Received for publication: 3 May 2018.

Revision received: 14 August 2018.

Accepted for publication: 27 August 2018.

This work is licensed under a Creative Commons Attribution 4.0 License (by-nc 4.0)

CCopyright T. Shoji et al., 2018

Licensee PAGEPress, Italy

Emergency Care Journal 2018; 14:7541

doi:10.4081/ecj.2018.7541

\section{Introduction}

Owing to the recent progress in technical skill sets and devices for intravascular treatment, arterial access procedures have significantly increased not only in the domain of percutaneous transluminal angioplasty (PTA) but also in emergency settings such as in performing arterial embolization and resuscitative endovascular balloon occlusion of the aorta (REBOA), in addition to intensive care settings requiring the use of invasive continuous hemodynamic monitoring tools such as PiCCO2 (PULSION Medical System, Germany) or EV1000 (Edwards Lifesciences, US) via a sheath placed in the femoral artery. Consequently, the occurrence of puncture site complications has also been increasing, and access-related complications can be fatal in a few cases. ${ }^{1-3}$ Therefore, emergency physicians and intensivists should be familiar with the risk factors associated with arterial access-related complications, the appropriate arterial access technique, particularly concerning accurate confirmation of the puncture site, and suitable treatment strategies to manage arterial accessrelated complications. In the current report, we evaluated the cause of critical hemorrhagic complications associated with femoral arterial access and the effectiveness of our treatment strategy utilizing a hybrid operation (endovascular treatment concomitant with surgical intervention) to manage a critical patient.

\section{Case Report}

A 40-year-old man had been prescribed an antiplatelet agent for left renal artery stenosis (RAS) secondary to Takayasu's arteritis. We performed PTA to his RAS via the left inguinal puncture approach without discontinuing the antiplatelet agent. Initially, a 6 Fr sheath was inserted into the left inguinal region; however, the procedure was technically difficult because the femoral artery was a narrow vessel, and the operator was not a specialist in radiology or vascular surgery and needed to perform multiple punctures. After the sheath was inserted, the patient was administered 3000 units of heparin intravenously. We then delivered the stent system into the left renal artery and deployed it. After angiography to confirm the patency of the renal artery and no complications following stent placement, the sheath was removed without reversal of heparinization, and we manually compressed the puncture site for $30 \mathrm{~min}$. The patient was placed on bed rest with a tampon gauze fixed to the puncture site for compression and pressure immobilization until the following morning. The following day, after puncture site decompression, he suddenly developed left-sided lower abdominal pain and shock (systolic blood pressure 80 $\mathrm{mmHg}$ and heart rate 120 beats/min), and his hemoglobin level dropped from 13.3 to $5.8 \mathrm{~g} / \mathrm{dL}$. Emergency computed tomography revealed leakage of the contrast agent from the left external iliac 
artery (EIA) and a ruptured pseudoaneurysm with a massive retroperitoneal hematoma. Based on PTA procedures, he was diagnosed with iatrogenic EIA trauma following high groin puncture (Figure 1), and an emergency operation was performed. Owing to the massive hematoma and active bleeding, we elected to use an endovascular technique prior to performing the surgical procedure. Under general anesthesia, we inserted a 5-Fr short sheath in the left superficial femoral artery via the retrograde access to make enough distance from the injury point for the interventional procedures (Figure 2A). We performed an angiography, and active bleeding was observed from the left EIA just above the origin of the left deep circumflex iliac artery medially (Figure 2B). We inserted a balloon catheter (MUSTANG $8 \mathrm{~mm} \times 4 \mathrm{~cm}$, Boston Scientific, U.S.), and controlled the hemorrhage by inflating the balloon over the bleeding point (Figure 2C). Subsequently, we exposed and confirmed the EIA via a retroperitoneal approach (Figure 3B-C). Although a massive hematoma was present, we could encircle the injured vessel and repair the puncture site safely and easily because the bleeding had initially been controlled following balloon occlusion (Figure 3D). We repeated the angiography through
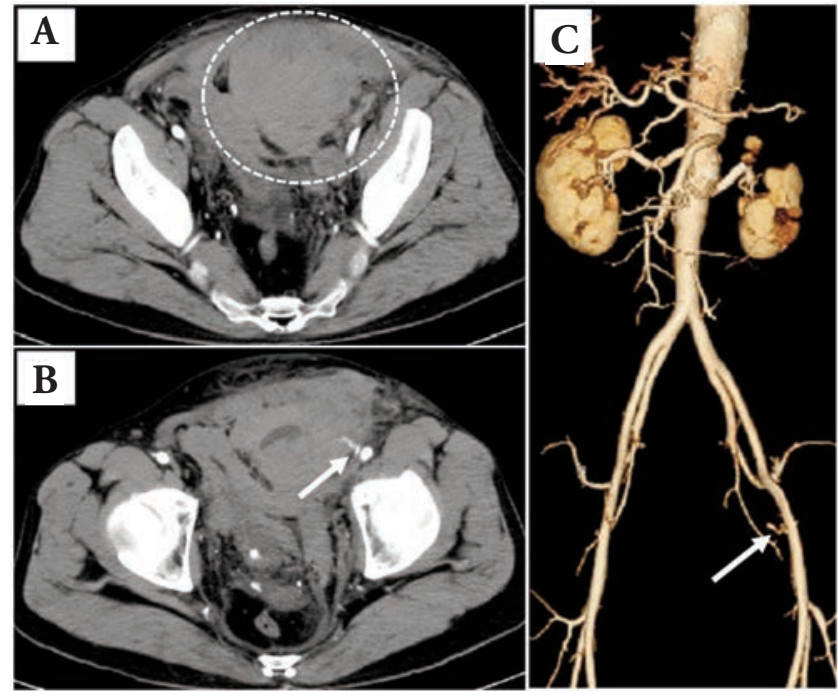

Figure 1. Preoperative computed tomography images. (A) Computed tomography (CT) shows a ruptured pseudoaneurysm associated with a massive retroperitoneal hematoma (dot-line circle); (B) CT shows leakage of the contrast agent from the left external iliac artery (EIA) (arrow); (C) three-dimensional computed tomography shows leakage of the contrast agent from the left EIA (arrow).
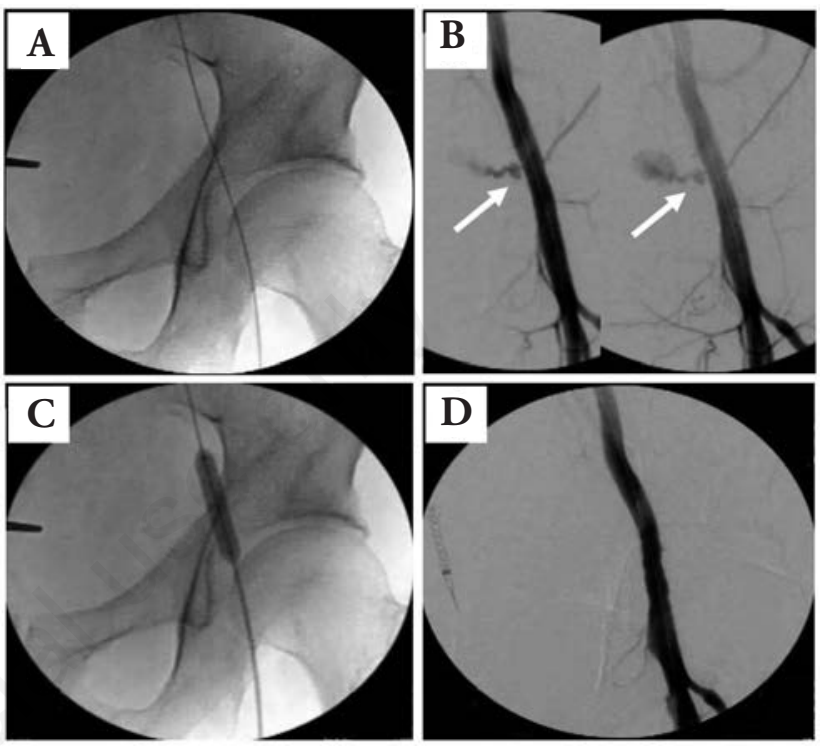

Figure 2. Intraoperative angiography images. (A) Image shows insertion of a $5 \mathrm{Fr}$ short sheath and guidewire into the left superficial femoral artery in a retrograde manner; (B) angiographic image shows a jet of blood spurting from the left external iliac artery just above the origin of the left deep circumflex iliac artery medially (arrow); (C) the balloon catheter (MUSTANG $8 \mathrm{~mm} \times 4 \mathrm{~cm}$, Boston Scientific, U.S.) is observed to have occluded the bleeding point; (D) an angiographic image of the completed operation.

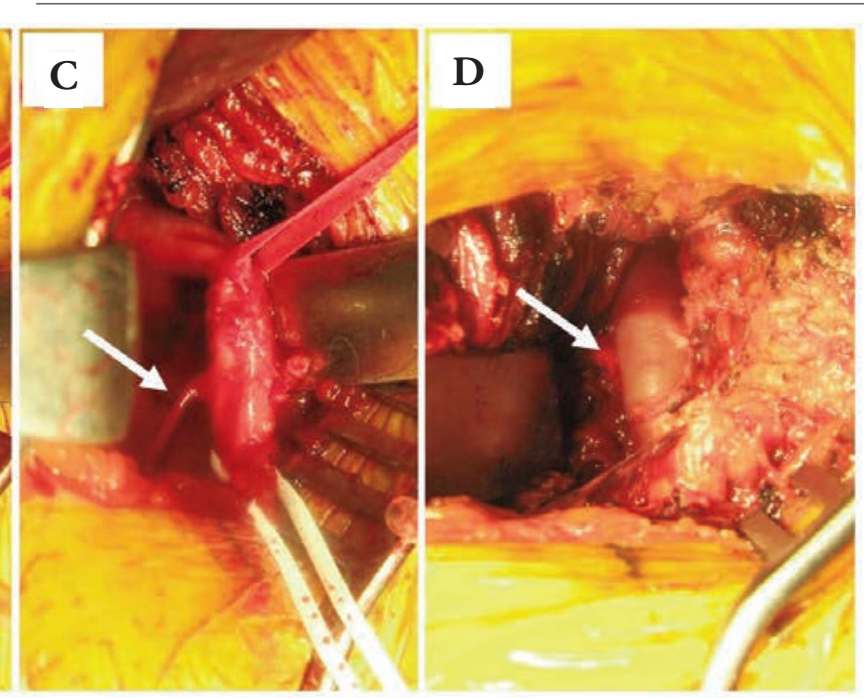

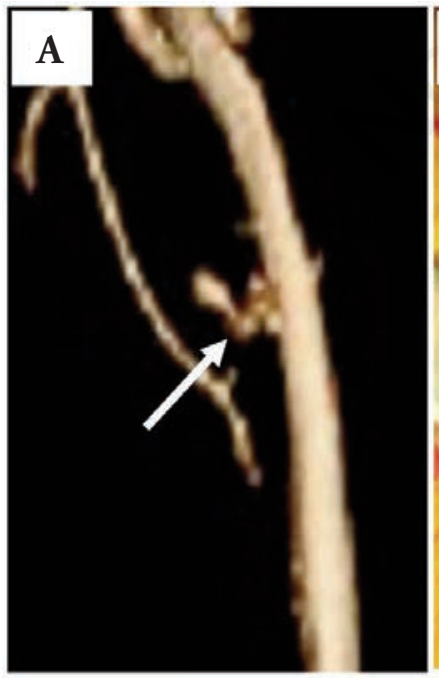

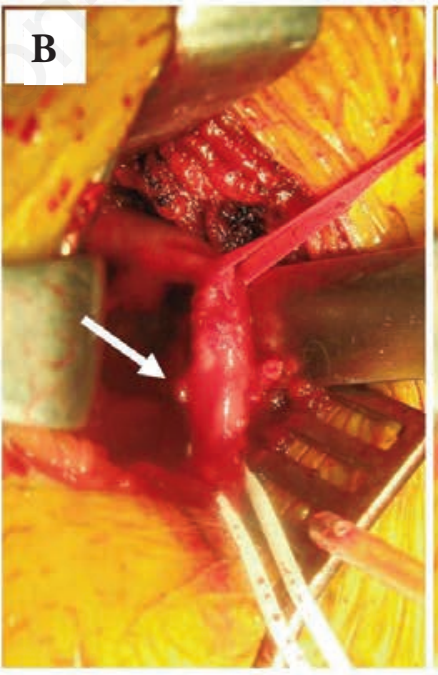

\section{.}


the sheath and confirmed that there was no further bleeding and stenosis (Figure 2D). Additionally, we surgically removed as much of the retroperitoneal hematoma as possible. The patient showed an uneventful postoperative course.

\section{Discussion}

Recently, emergency physicians and intensivists have been commonly performing percutaneous arterial access procedures, such as percutaneous arterial embolization, REBOA, and the use of invasive continuous hemodynamic monitoring tools. Therefore, instances of iatrogenic vascular trauma related arterial access have also increased. Previous studies describing pseudoaneurysms complicating arterial access procedures have reported rates ranging between 0.2 and $8 \%$. $^{1,2,4-7}$

Risk factors for iatrogenic arterial injury include: i) patientrelated factors such as age ( $>70$ years), female gender, obesity, severe arteriosclerosis, hypertension, congestive heart failure, renal dysfunction (creatinine clearance $<60 \mathrm{~mL} / \mathrm{min}$, or patients undergoing hemodialysis), coagulopathy, and an anatomical variant showing high branching of the femoral artery, and ii) medical procedure-related factors including use of antithrombotic therapy, inadequate knowledge of catheterization, use of a large-sized sheath/catheter ( $>8 \mathrm{Fr}$ ), an inappropriately performed catheterization (multiple punctures, high/low puncture, and poor handling of devices), and inadequate compression methods at the puncture site. ${ }^{1-6}$ Regarding an optimal puncture technique, the common femoral artery (CFA) must be safely punctured in the groin after careful confirmation of the anatomy. Because the caput femoris is located behind the CFA, it is possible to compress the puncture site effectively and safely, thereby ensuring a lower possibility of pseudoaneurysm and hematoma formation. Although physicians tend to puncture the inguinal skin folds of the groin for an anatomical guide, complications may be observed depending upon the patient's body type/habitus. Therefore, accurate confirmation of the puncture site under fluoroscopic- or ultrasonographic guidance can minimize the incidence of complications. Kodera examined the appropriate femoral artery puncture site based on the anatomical confirmation using radiological imaging and reported that the femoral artery should be punctured at the level of the lower fourth of the femoral head. ${ }^{8}$ In our patient, we concluded that adequate compression and hemostasis could not be achieved owing to the inadequate procedure, especially high EIA puncture, without discontinuation of the antiplatelet agent. As a result, pseudoaneurysm on the EIA was formed, and pseudoaneurysm ruptured into the retroperitoneal space because of body movements after the pressure was released.

Small non-ruptured pseudoaneurysms measuring $<2.0-3.0 \mathrm{~cm}$ in diameter tend to thrombose spontaneously within 4-6 weeks without requiring antithrombotic therapy. ${ }^{2,4-6,9}$ Several embolization methods have been described in previous reports, such as compression of the vascular trauma site using a probe device under ultrasonographic guidance, ${ }^{1,2,4-7,9}$ puncturing the pseudoaneurysm and injecting thrombin ${ }^{1,2,4-6,9,10}$ or N-butyl-2-cyanoacrylate percutaneously into the pseudoaneurysm, ${ }^{11}$ or endovascular coil embolization. ${ }^{4,6,12}$ However, these treatment strategies are ineffective in managing ruptured pseudoaneurysms because these methods are not completely safe and useful particularly in patients who are hemodynamically unstable. In addition, for some vascular trauma cases, the use of stent graft is effective. ${ }^{1,4-6}$ However, because active bleeding was observed from the left EIA, just above DCIA in present case, we must place the stent on the hip joint to cover the injury point. As a stent placed on the hip joint could cause stent fracture or occlusion due to joint movement, we could not use a stent graft system. Therefore, in patients with ruptured pseudoaneurysms, unstable hemodynamics, and an unsuitable case for the use of stent graft system, surgical repair of the injured blood vessel is recommended as the safest and most effective method of management. However, when a large hematoma is present as was observed in our patient, and when the injured vessel is located in a deep and not easily accessible site, such as in the retroperitoneum, it is difficult to directly access to the injured site. This causes continued bleeding and prolongs the operative time because of the difficulty with access and often the inability to obtain a clear and effective surgical field. ${ }^{1}$ However, an endovascular procedure can provide safe and easy access to the injured vessel via healthy vessels used as access points. Using this method, we can confirm the injured site angiographically, promptly perform primary hemostasis using balloon occlusion, and repair the injured artery easily and safely. Moreover, it is helpful that we can obtain an angiographic image of the completed surgery to confirm that there was no further bleeding and stenosis. Additionally, in the cases with the massive retroperitoneal hematoma, surgical removal of the hematoma is important to prevent secondary neurovascular complications such as distal neuropathy and deep venous thrombosis following compression caused by the massive hematoma. ${ }^{3}$

\section{Conclusions}

Understanding possible risk factors and accurate confirmation of puncture sites are important to reduce the risk of iatrogenic arterial access-related complications. Moreover, emergency physicians and intensivists who perform arterial access should be familiar with the optimal emergency treatment that would be required to control/manage arterial access-related complications. In addition, in patients with critical hemorrhagic complications, a hybrid operation should be considered to ensure a reliable and safe treatment.

\section{References}

1. Tsetis D, Perdikakis E, Kehagias E. Percutaneous treatment if iatrogenic femoral artery access complications. Hell J Radiol 2017;2:51-65.

2. Kolluri R, Fowler B, Nandish S. Vascular access complications: diagnosis and management. Curr Treat Options Cardiovasc Med 2013;15:173-87.

3. Sreeram S, Lumsden AB, Miller JS, et al. Retroperitoneal hematoma following femoral arterial catheterization: a serious and often fatal complication. Am Surg 1993;59:94-8.

4. Morgan R, Belli AM. Current treatment methods for postcatheterization pseudoaneurysms. J Vasc Interv Radiol 2003; 14:697-710.

5. Tsetis D. Endovascular treatment of complications of femoral arterial access. Cardiovasc Intervent Radiol 2010;33:457-68.

6. Ahmad F, Turner SA, Torrie P, Gibson M. Iatrogenic femoral artery pseudoaneurysms-a review of current methods of diagnosis and treatment. Clin Radiol 2008;63:1310-6.

7. Coley BD, Roberts AC, Fellmeth BD, et al. Postangiographic femoral artery pseudoaneurysms: further experience with USguided compression repair. Radiology 1995;194:307-11.

8. Kodera K. Radiologic-anatomical study of femoral artery 
puncture site for percutaneous angiography [in Japanese with English abstract]. Keioigaku 1999;76:179-86.

9. Heis HA, Bani-Hani KE, Elheis MA, et al. Postcatheterization femoral artery pseudoaneurysms: therapeutic options. A casecontrolled study. Int J Surg 2008;6:214-9.

10. Paulson EK, Sheafor DH, Kliewer MA, et al. Treatment of iatrogenic femoral arterial pseudoaneurysms: comparison of US-guided thrombin injection with compression repair.
Radiology 2000;215:403-8.

11. Aytekin C, Firat A, Yildirim E, et al. Ultrasound-guided glue injection as alternative treatment of femoral pseudoaneurysms. Cardiovasc Intervent Radiol 2004;27:612-5.

12. Lemaire JM, Dondelinger RF. Percutaneous coil embolization of iatrogenic femoral arteriovenous fistula or pseudoaneurysm. Eur J Radiol 1994;18:96-100. 